\title{
The modulation of neural blood pressure control by blood pressure pulsatility
}

\author{
Hasana Bagnall Hare \\ Dept. of Electronic Engineering \\ Maynooth University \\ Maynooth, Co. Kildare, Ireland \\ hasana.bagnallhare.2016@mumail.ie
}

\author{
John V. Ringwood \\ Dept. of Electronic Engineering \\ Maynooth University \\ Maynooth, Co. Kildare, Ireland \\ john.ringwood@mu.ie
}

\begin{abstract}
The neural control of blood pressure involves effectors related both to peripheral resistance and the heart. These mechanisms, using blood pressure measurements from the baroreceptors, work in unison to maintain blood pressure at an acceptable value. However, it is less well known that the neural baroreflex is affected by the pulsatile nature of blood flow, which also modulates the blood pressure signal. Furthermore, the presence, or absence, of low-frequency (circa $0.1 \mathrm{~Hz}$ in humans) oscillations in blood pressure may be predicated on the pulsatility, or lack of pulsatility. The absence of pulsatility may be found in patients with artificial (turbine type) hearts, or with left ventricular assist devices (LVADs). This paper shows the effect of pulsatility on the neural baroreflex, and consequently on the generation of low-frequency oscillations, via simulation results.

Index Terms-Feedback control, limit cycle, baroreflex, neural control, low-frequency oscillations, pulsatility
\end{abstract}

\section{INTRODUCTION}

The neural baroreflex has been studied through a number of disciplines, and there is general acceptance of a neural feedback mechanism via the baroreceptors and the brain [1]. In the baroreflex, shown in Fig.1, blood pressure (BP) is measured via the baroreceptors (in the aortic arch and carotid sinus) and actuated via manipulation (via sympathetic nerves) of smooth muscle in the arterioles, and via manipulation of cardiac output (via sympathetic and parasympathetic nerves).

Over the last few decades, there has been increasing interest in low frequency (LF) oscillations in blood pressure, often termed Mayer waves [2], occurring at around $0.1 \mathrm{~Hz}$ in humans. While their purpose is uncertain, and their origin not yet agreed upon, certain evidence for their origin as a result of a neural baroreflex nonlinearity has been given [3], where the oscillation frequency has been shown to scale well with variation in species.

A final aspect related to the work reported in this paper is that of blood pressure pulsatility. In the vast majority of cases, analysis of the neural baroreflex omits any consideration of blood pressure pulsatility, and instead considers some heartperiod averaged quantity, such as mean arterial pressure (or perhaps systolic, or diastolic). In particular, there is a paucity

The authors are grateful for the experimental blood pressure data provided by Dr. S.-J. Guild of the Dept. of Physiology at Auckland University.

978-1-7281-2800-9/19/\$31.00 @2019 IEEE of research on what the effect a lack of pulsatility might have on the baroreflex. While it could be argued that this is an unimportant case due to the fact that all humans and animals have pulsatile hearts, interventions such as (turbine-based) artificial hearts, or left ventricular assist devices (LVADs), may diminish, or completely extinguish, blood pressure pulsatility.

This paper enumerates the effect of a disappearance of pulsatility on the baroreflex via both an analytical technique and simulation, and shows that the presence of pulsatility acts to reduce the neural baroreflex gain. This gain reduction, or increase in the absence of pulsatility, may have some long-term physiological effects [4], [5], but such discussion is beyond the scope of this paper. However, this study does quantify the potential effect of a change in pulsatility on LF BP oscillations, where some experimental evidence exists that LVAD insertion results in an increase in Mayer wave activity which, in turn, can be linked with an increase in baoreflex gain [3], [6].

The original contributions of this paper may be summarised as follows: A complete (peripheral resistance + cardiac branches) baroreflex model is presented, with the effect of pulsatility on the complete baroreflex presented, while the effect only on the peripheral resistance branch was considered in [6]. Finally, a higher fidelity blood pressure trace is now used to evaluate a piecewise linear approximation to the periodic pulsatile $\mathrm{BP}$ signal, as required in the equivalent nonlinearity (EQNL) calculation [7] (see Section IV-A). The paper therefore allows the effect of pulsitility on the complete neural baroreflex to be evaluated, which manifests itself in terms of modulation of low-frequency oscillations in mean arterial pressure (MAP), as a result of changes in baroreflex gain.

\section{THE NEURAL BAROREFLEX}

The baroreflex is the homeostatic mechanism that maintains blood pressure. The neurally controlled baroreflex model used in Fig.1 shows the critical components, which include: dynamic components $G(s)$, time delays $\tau$ and non linearities $f(s)$. The model in fig. 1 is a three branch model: parasympathetic cardiac, sympathetic cardiac and sympathetic resistance, operating as a feedback system to maintain a $\mathrm{BP}$ set point. The main controller for the baroreflex is considered to be the central nervous system (CNS) as depicted in Fig.1. 


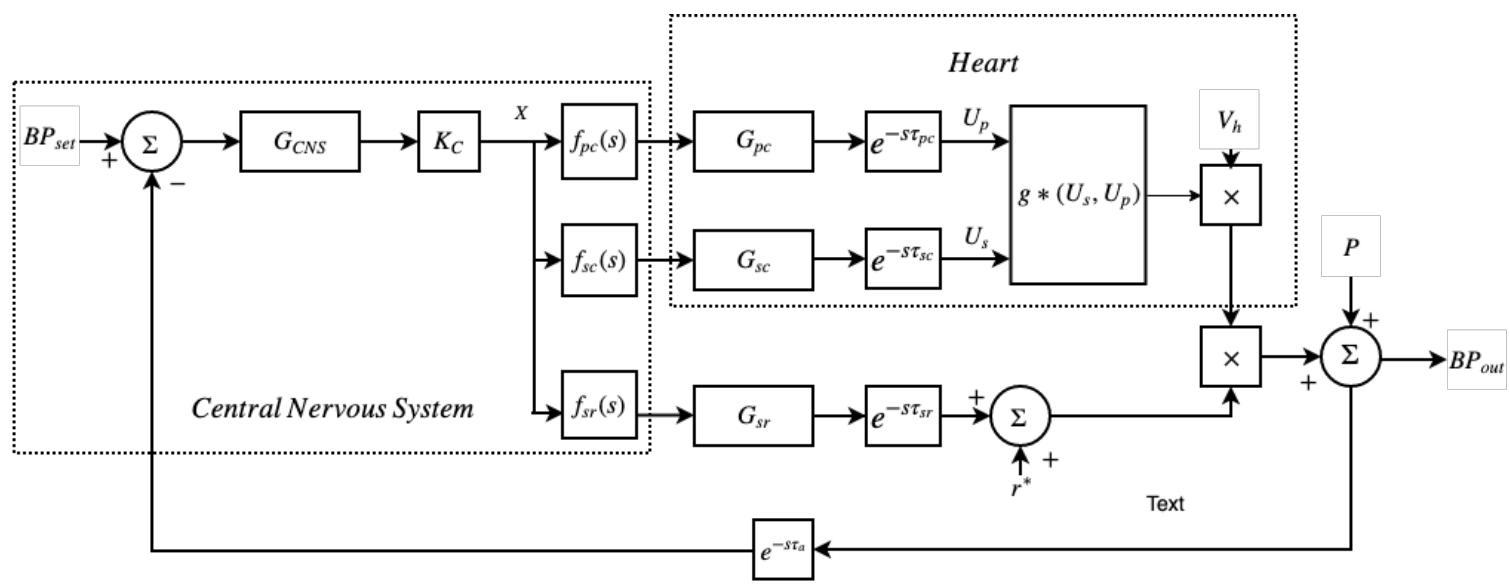

Fig. 1. The neural baroreflex, containing both peripheral resistance and cardiac branches. $P$ denotes blood pulsatility, represented as a pressure signal

The CNS accepts sensory measurements of blood pressure from baroreceptors in the aortic arch and carotid sinus, and influences blood pressure primarily through manipulation of peripheral resistance (via neurally innervated smooth muscles around the arterioles) and heart-rate. The dynamic components of the model are expressed as:

$$
\begin{gathered}
G_{C N S(s)}=e^{-s \tau_{b} \frac{1.33 s+1}{s+1}} \\
G_{p c(s)}=k_{p c} \frac{1}{1.22 s+1} \\
G_{s c(s)}=k_{s c} \frac{1}{1.29 s^{2}+1.29 s+1} \\
G_{s r(s)}=k_{s r} \frac{11 s^{2}+6.64 s+1}{4.27 s^{4}+21 s^{3}+36 s^{2}+22 s+1}
\end{gathered}
$$

All other quantities are defined below:

- $r^{*}$ vascular resistance of arteries

- $V_{h}$ stroke volume of the heart

- $f h$ heart rate

- $B_{p}^{\text {set }}$ blood pressure set point

- $B_{p}^{o u t}$ resulting blood pressure

- $e^{-s \tau}$ delay introduced by smooth muscle and nerves at various stages throughout the model. The distinct time delays are shown in table I

The nonlinearities $(f(s))$ represent the baroreflex curve, which is expressed as an arctan function:

$$
y=h \tan ^{-1}\left(\beta\left(x-x^{*}\right)\right)+y^{*}
$$

The cardiac branches are combined through the following equation:

$$
g *\left(U_{p}, U_{s}\right)=k_{p} U_{p}+k_{s} U_{s}+f_{h}
$$

where, $k_{s}=0.88$, sympathetic gain, $k_{p}=-1.2$

The arctan function in equation 5 was parameterised for the three branches of the baroreflex system: parasympathetic cardiac, sympathetic cardiac and sympathetic resistance. The parameters shown in table II were found in [6] to fit experimental data for rabbits experiencing normoxia. When accounting for peripheral resistance, the model ignores arterial compliance and has a constant $\left(r^{*}\right)$ for vascular resistance.

TABLE I

Time Delays For Baroreflex Model

\begin{tabular}{lllll}
\hline$\tau_{p c}$ & $\tau_{s c}$ & $\tau_{s r}$ & $\tau_{a}$ & $\tau_{b}$ \\
\hline 0.3 & 0.8 & 0.85 & 0.2 & 0.4 \\
\hline
\end{tabular}

TABLE II

ARCTAN PARAMETERS FOR NORMOXIA

\begin{tabular}{lllll}
\hline Arctan function & $x^{*}$ & $y^{*}$ & $\beta$ & $h$ \\
\hline Original Arctan Function & -71 & 55 & -0.12 & 33 \\
\hline
\end{tabular}

\section{LOW-FREQUENCY OSCILLATIONS}

Low-frequency (LF) oscillations, often called Mayer waves [2] occur at frequencies below respiration and heart rate, typically at around $0.1 \mathrm{~Hz}$ in humans and $0.3 \mathrm{HZ}$ in rabbits. The LF oscillation frequency is dependent on species size and this dependence has been explained by a nonlinear feedback model [3], with the length of the nerve conduction paths (i.e. conduction delay) being responsible for the frequency variation

The development in [3], which only considers the peripheral resistance control of BP, uses an extension of Nyquist's stability criterion, via a describing function representation of the baroreflex curve [8]. However, Nyquist's criterion cannot be directly applied to the full neural BP control system, consisting of both cardiac and peripheral resistance effectors, as shown in Fig.1. Rather, an alternative approach, based on establishing conditions for sustained propagation around the neural BP control loop is required. [9]

However, both approaches (Nyquist and sustained propagation) require a compact representation of the nonlinear baroreflex curves representing the static relationship between 
BP and sympathetic/parasympathetic nerve activity to the peripheral resistance and heart. This can be achieved via the describing function [8].

\section{A. Describing function representation}

While the sigmoidal baroreflex curves can be represented by a range of parametric functions, the arctan function has been shown to be a good fit and leads, with some manipulation, to a relatively simple describing function representation [8]. Specifically, for the arctan function in Equation (5), the describing function is given [8] as:

$$
D F(M)=\frac{2 h}{\beta M^{2}}\left(\sqrt{1+\beta^{2} M^{2}}-1\right)
$$

where $\beta$ and $h$ represent the parameters of the arctan function (see Table II) and $M$ is the effective amplitude of the sinusoidal oscillation incident on the arctan function. We note that one of the assumptions of the describing function representation is that any harmonics of the incident sinusoid (sustained oscillation), appearing at the nonlinearity output, are effectively eliminated by the low-pass filtering characteristics of following dynamical elements. This is ensured by the low-pass nature of the dynamic blocks $G_{p s}, G_{s c}$, and $G_{r c}$, which follow the nonlinear characteristics $f_{p c}, f_{s c}$, and $f_{r c}$, respectively.

\section{B. Factors affecting LF oscillations}

There are a number of physiological conditions and interventions which have been shown to affect the presence/absence of LF BP oscillations. These include hypoxia [8], Haemorrhage [10], and modulation of nitric oxide synthesis [11]. Invariably, these interventions involve modulation of the overall baroreflex gain, which is consistent with the nonlinear feedback theory for LF oscillations in [3].

However, one phenomenon which has the potential to alter baroreflex gain, and consequently affect the presence/absence of LF oscillations, but has received relatively little exposure in the literature, is BP pulsatility. BP pulsatility may be modulated in cases where cardiac flow assist devices, such as LVADs or turbine-based artificial hearts. Some evidence exists [12] that a reduction in pulsatility is accompanied by an increase in the incidence of LF oscillations. The specific focus of the current research is to establish the link between pulsatility, baroreflex gain and the presence/absence of LF oscillations as a surrogate measure of baroreflex gain.

\section{EFFECT OF PULSATILITY}

The effect of pulsatility on the baroreflex system will be investigated in two ways: firstly, where a pulsatile signal is introduced, and another where the baroreflex parameters are modified (via an 'equivalent nonlinearity') to represent the effect of pulsatile blood pressure signals. A pulsatile signal, $P$, derived from the blood pressure signal shown in Fig. 3, is introduced into the model in Fig.1.The effect pulsatility has on modulating baroreflex curves, $f_{p c}, f_{s c}$, and $f_{s r}$, is also considered as described in Section IV-A.

\section{A. Equivalent nonlinearity}

The EQNL concept used, based on the approach outlined in [7], is illustrated in fig. 2, which allows a nonlinear function, having an input consisting of a low-frequency signal, together with a high-frequency (in this case, pulsatile) signal, to be represented by a new nonlinear function (the equivalent nonlinearity) having only the low-frequency signal as the input.

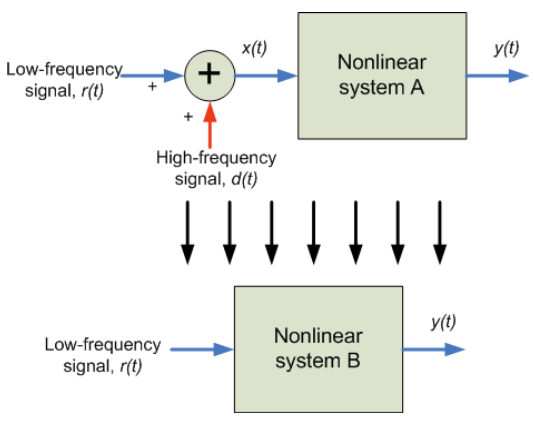

Fig. 2. EQNL concept

The first step in obtaining the equivalent nonlinearity is to represent the pulsatile signal with a piecewise linear approximation, as shown in shown in Fig. 3.

The signal in figure 3 comes from a set of clinical data measuring blood pressure from the abdominal aorta, One complete cycle was segmented into 4 parts, $t_{0}-t_{1}, t_{1}-t_{2}$, $t_{2}-t_{3}, t_{3}-t_{p}$, in a similar manner to that done in [6] and [13], though the pulsatile data used for the current approximation is of significantly higher resolution. The parameters for the individual segments of the piecewise linear approximation are shown in Table. III

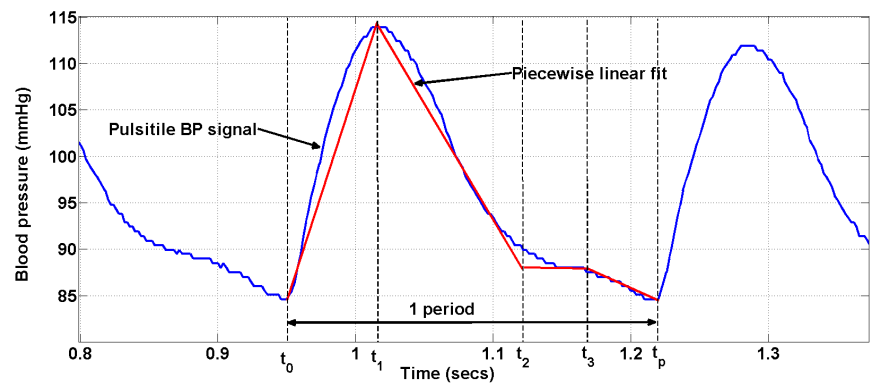

Fig. 3. Piecewise Rabbit BP signal

TABLE III

SEgmentation of Clinical Data

\begin{tabular}{lllll}
\hline Parameter & $t_{o}-t_{1}$ & $t_{1}-t_{2}$ & $t_{2}-t_{3}$ & $t_{3}-t_{p}$ \\
\hline$A$ (height) & 29.18 & 26.02 & 0 & 3.37 \\
$\tau$ (length) & 0.068 & 0.104 & 0.0452 & 0.053 \\
\hline
\end{tabular}

For a candidate nonlinearity $f($ ), the original nonlinear system's output is:

$$
y=f(r+d(t))
$$


Let $p(q) d q$ be the probability that, for any time $t, d(t)$ lies in the range $q$ to $q+d q$. When $d(t)$ has the value $q$, the expected value for $y$ is [6]:

$$
\bar{y}=\int_{-\infty}^{\infty} f(r+q) p(q) d q
$$

To calculate $p(q)$, let $F(q)$ be the probability that the dither signal is above $q$, giving

$$
\frac{d F(q)}{d q}=-p(q)
$$

Three of the four segment are triangles with an offset of $b$ and an amplitude $A$, this results in the probability density function being expressed as:

$$
p(q)=-\frac{d F(p)}{d p}=\frac{1}{2 A}
$$

The equivalent nonlinearity (EQNL) for the first segment $t_{0}-t_{1}$ is found by:

$$
\overline{y_{1}}=\int_{b+A_{1}}^{b-A_{1}} \frac{1}{2 A_{1}}\left[h \tan ^{-1}(\beta(r+q))+y^{*}\right] d q
$$

Both the second and fourth segments are triangles and were found in a similar manner to the first segment. The probability density function uses the values in table III. The third segment however is a straight like with an offset of b. Meaning the linearity is the original EQNL but with an offset of $b$ and a constant probability function:

$$
\left.\overline{y_{3}}=h \tan ^{-1}(\beta(r+q))+y^{*}\right] d q
$$

The final EQNL for the dither signal is the combination of all four segments:

$$
\bar{y}=\alpha_{1} \overline{y_{1}}+\alpha_{2} \overline{y_{2}}+\alpha_{3} \overline{y_{3}}+\alpha_{4} \overline{y_{4}}
$$

where $\alpha_{1}=0.252, \alpha_{2}=0.385, \alpha_{3}=0.167, \alpha_{4}=0.196$.

\section{B. Approximate model}

The approximate model is the model shown in fig. 1 where $P=0$, but the nonlinearities have the EQNL baroreflex parameters. To find these parameters we graph the original arctan function in equation 5 beside the EQNL. We then alter the slope $(B)$ of equation 5 to match the gain and midpoint of the EQNL. This is carried out three times one for each of the nonlinearities $(f(s))$ in fig. 1 . The altered $B$ is shown in table IV.

TABLE IV

$B$ VALUES FOR BAROREFLEX BRANCHES

\begin{tabular}{llll}
\hline Arctan Function & $B_{p c}$ & $B_{s c}$ & $B_{s r}$ \\
\hline Original Arctan (Case 1\&2) & -0.1342 & 0.02 & 0.0383 \\
EQNL Arctan (Case 3) & $-0.1342 * 0.75$ & $0.02 * 0.75$ & $0.383 * 0.45$ \\
\hline
\end{tabular}

The modified arctan parameters are deemed a fit to the EQNL if the midpoint and the slope $(B)$ 's of both match. This is the case for all three branches:

- Fig.4 shows the original baroreflex curve for the parasympathetic cardiac branch of the baroreflex $\left(f_{p c}(s)\right)$, the equivalent nonlinearity obtained by including the pulsatile BP input, and the best arctan fit to the EQNL curve. The fit shows a $25 \%$ reduction in slope $(B)$.

- Fig.5 shows the original baroreflex curve for the sympathetic cardiac branch of the baroreflex $\left(f_{s c}(s)\right)$, the equivalent nonlinearity obtained by including the pulsatile $\mathrm{BP}$ input, and the best arctan fit to the EQNL curve. The fit shows a $25 \%$ reduction in slope $(B)$.

- Fig.6 shows the original baroreflex curve for the sympathetic resistance branch of the baroreflex $\left(f_{s r}(s)\right)$, the equivalent nonlinearity obtained by including the pulsatile BP input, and the best arctan fit to the EQNL curve. The fit shows a $55 \%$ reduction in slope $(B)$.

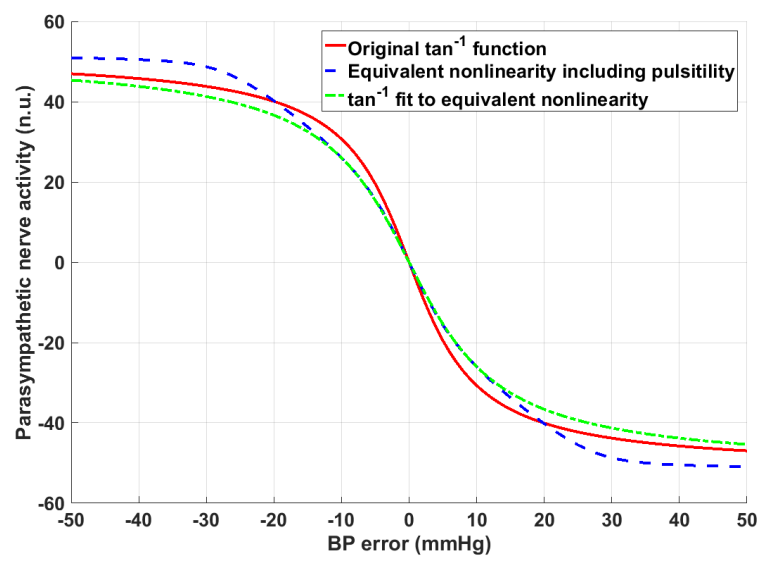

Fig. 4. Parasympathetic Baroreflex gain altered by presence of pulsatility

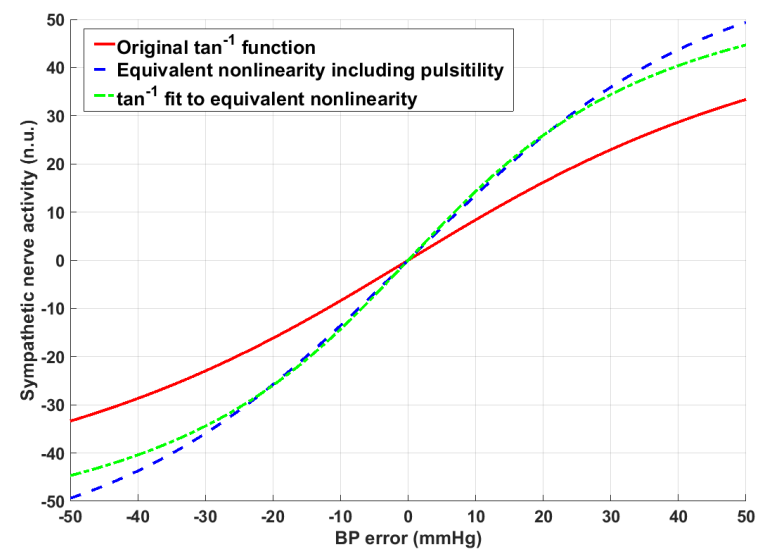

Fig. 5. Sympathetic Baroreflex gain altered by presence of pulsatility 


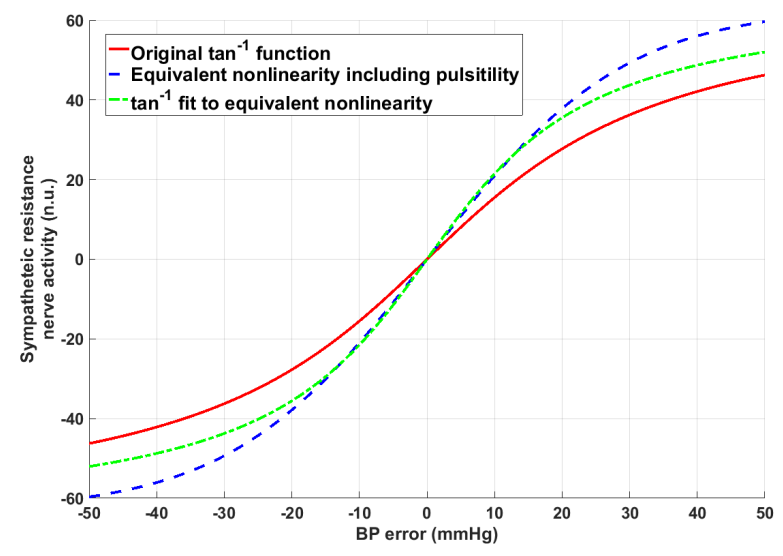

Fig. 6. Sympathetic Resistance Baroreflex gain altered by presence of pulsatility

TABLE V

TEST CONDITIONS

\begin{tabular}{lll}
\hline Case & Baroreflex Gain & Pulse Signal \\
\hline 1 & Original Arctan Function Baroreflex Parameters & Yes \\
2 & Original Arctan Function Baroreflex Parameters & No \\
3 & EQNL Baroreflex Parameters & No \\
\hline
\end{tabular}

\section{RESUlts}

The effect of pulsatility on the neural baroreflex was investigated by comparing three cases of the model shown in fig.1. The test conditions for all three cases are a combination of the arctan parameters from table IV and the presence or absence of the pulsatile signal $P$. The conditions for each case are shown in the table $\mathrm{V}$.

When testing the three cases, the gain value for the $K_{C}$ block in 1 is set to investigate both the presence and absence of LF oscillations. The gain of the CNS is established through a trial basis as a physiological value is hard to acquire due to the difficulty in obtaining an absolute measure of nerve activity.

The results from the 3 cases detailed in Table $\mathrm{V}$ may be described as follows: case 2 is the first to display LF oscillations this is the only case that does not account for a pulsating heart, either via the EQNL baroreflex parameters, or injection of the pulse signal $P$ itself as shown in Fig.7, while cases 1 and 3 both remain at a steady state value (no LF oscillations). Note also that the pulsatile (high-frequency) is not obvious on the BP signals in Fig.7.

Fig.7 shows that a lack of pulsatile behaviour increases the baroreflex's sensitivity to LF oscillations. This is due to the effective increase in baroreflex gain; note the greater gain (slope) of the baroreflex curves for the non-pulsatile cases in Figs.4, 5, and 6.

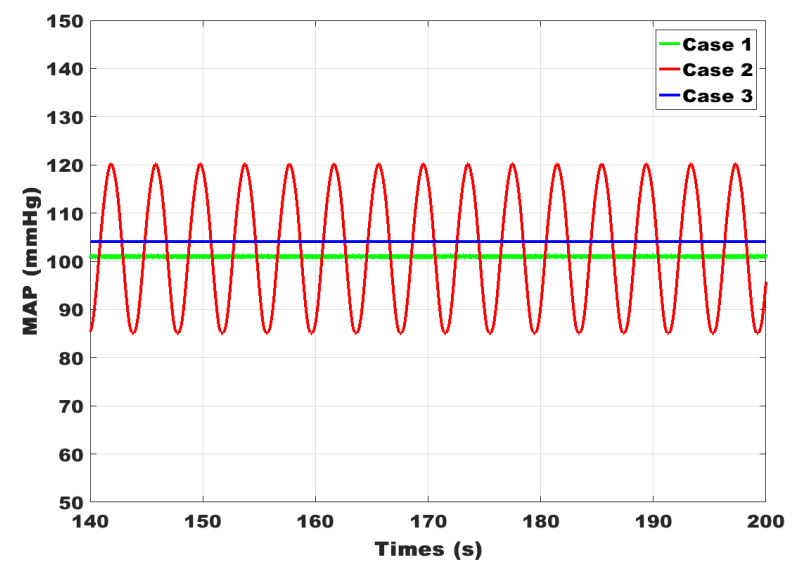

Fig. 7. CNS gain with case 2 oscillating

\section{CONCLUSIONS}

A lack of pulsatility can lead to elevation of the baroreflex gain which, in turn, can manifest itself via an increase in the likelihood of LF oscillations. While an increase in the presence of LF oscillations may, in itself, be relatively unimportant (though symptomatic of elevated baroreflex gain), it may have other more profound consequences. To date, little is understood regarding any effects that LVADs, or artificial (turbine type) hearts may have on the neural baroreflex. This paper demonstrates that elevated baroreflex gain ensues with a decrease in blood flow/pressure pulsatility, which may have longer term effects.

\section{REFERENCES}

[1] S. Malpas, B. Leonard, S.-J. Guild, J. Ringwood, M. Navakatikyan, P. Austin, G. Head, and D. Burgess, "The role of the sympathetic nervous system in regulating blood pressure variability," IEEE Engineering in Medicine and Biology Magazine, vol. 20, pp. 17-24, 2001.

[2] C. Julien, "The enigma of mayer waves: facts and models," Cardiovascular research, vol. 70, no. 1, pp. 12-21, 2006.

[3] J. V. Ringwood and S. C. Malpas, "Slow oscillations in blood pressure via a nonlinear feedback model," American Journal of PhysiologyRegulatory, Integrative and Comparative Physiology, vol. 280, no. 4 , pp. R1105-R1115, 2001.

[4] N. Moazami, W. P. Dembitsky, R. Adamson, R. J. Steffen, E. G. Soltesz, R. C. Starling, and K. Fukamachi, "Does pulsatility matter in the era of continuous-flow blood pumps?" Journal of Heart and Lung Transplantation, vol. 4, no. 8, pp. 999-1004, 2015.

[5] T. Drews, M. Jurmann, D. Michael, P. Miralem, Y. Weng, and R. Hetzer, "Differences in pulsatile and non-pulsatile mechanical circulatory support in long-term use," The Journal of Heart and Lung Transplantation, vol. 27, no. 10, pp. 1096-1101, 2008.

[6] J. V. Ringwood, F. Taussi, and A. M. de Paor, "The effect of pulsatile blood flow on blood pressure regulatory mechanisms," in 2012 IEEE International Conference on Control Applications. IEEE, 2012, pp. 609-614.

[7] R. Simpson and H. Power, "Applications of high frequency signal injection in non-linear systems," International Journal of Control, vol. 26, no. 6, pp. 917-943, 1977.

[8] A. de Paor and J. Ringwood, "A simple soft limiter describing function for biomedical applications," IEEE transactions on biomedical engineering, vol. 53, no. 7, pp. 1233-1240, 2006. 
[9] J. Ringwood and O. K. S. Malpas, "Prediction of low frequency blood pressure oscillations via a combined heart/resistance model," IFAC Proceedings Volumes, vol. 38, no. 1, pp. 60-65, 2005.

[10] S. C. Malpas, R. G. Evans, G. A. Head, and E. V. Lukoshkova, "Contribution of renal nerves to renal blood flow variability during hemorrhage," American Journal of Physiology-Regulatory, Integrative and Comparative Physiology, vol. 274, no. 5, pp. R1283-R1294, 1998.

[11] M. Castellano, D. Rizzoni, M. Beschi, M. L. Muiesan, E. Porteri, G. Bettoni, M. Salvetti, A. Cinelli, R. Zulli, and E. Agabiti-Rosei, "Relationship between sympathetic nervous system activity, baroreflex and cardiovascular effects after acute nitric oxide synthesis inhibition in humans." Journal of hypertension, vol. 13, no. 10, pp. 1153-1161, 1995.

[12] R. L. Cooley, N. Montano, C. Cogliati, P. Van de Borne, W. Richenbacher, R. Oren, and V. K. Somers, "Evidence for a central origin of the low-frequency oscillation in rr-interval variability," Circulation, vol. 98, no. 6, pp. 556-561, 1998.

[13] M. Loughman, A. de Paor, and J. V. Ringwood, "The effect of blood pressure pulsatility on the baroreflex gain," in 2016 27th Irish Signals and Systems Conference (ISSC). IEEE, 2016, pp. 1-6. 